\title{
Simultaneous recording of cerebrospinal fluid pressure and middle cerebral artery blood flow velocity in patients with suspected symptomatic normal pressure hydrocephalus
}

\author{
D W Droste, J K Krauss
}

\begin{abstract}
CSF pressure (intracranial pressure, in one patient lumbar pressure) was monitored continuously for one night in 23 patients with suspected symptomatic normal pressure hydrocephalus (NPH) to identify patients who might benefit from subsequent shunt surgery. In 20 patients middle cerebral artery (MCA) blood flow velocity by means of transcranial Doppler sonography (TCD) and CSF pressure were recorded simultaneously. In three patients transcranial Doppler signals were insufficient. Spontaneous changes in CSF pressure always paralleled changes in the TCD signal. Equivalents of B-waves as well as intermediate waves (in between Band $A$-waves), and $C$-waves could be identified easily and always appeared in phase. The Doppler signal, however, could not be used to evaluate the absolute changes in CSF pressure. Fast Fourier Transform of the Doppler signal was a useful tool to indicate the relative frequency of $B$-wave equivalents. In five patients the injection of $10 \mathrm{ml}$ saline into the ventricle raised intracranial pressure considerably, but hardly affected the MCA blood flow velocity. Continuous TCD monitoring might be useful as a noninvasive screening procedure in patients with suspected symptomatic NPH before continuous invasive CSF pressure measurements are performed.
\end{abstract}

$(\Im$ Neurol Neurosurg Psychiatry 1993;56:75-79)

A frequent problem encountered in neurology and neurosurgery is determining which patients with suspected normal pressure hydrocephalus (NPH) will improve clinically after shunt surgery. Positive criteria for the diagnosis of NPH include gait disturbance, dementia and urinary incontinence, periventricular lucency and large ventricles demonstrated on CT, and clinical improvement after lumbar puncture. Overnight recording of intracranial pressure (ICP) has become an established method for selecting patients who will respond to shunt surgery.

Clinical improvement has been correlated positively with recordings of $\mathrm{A}$-waves or with frequent high amplitude B-wave activity. ${ }^{1-4}$ B-waves are spontaneous ICP oscillations with a wave length of $0.5-2$ minutes. A-waves last longer, have a higher amplitude, and appear plateau-like (therefore also referred to as "plateau waves"). Lundberg also described C-waves particularly on the plateaux of the A-waves. ${ }^{5}$ B-waves are found with impaired CSF absorption in NPH and also less frequently in healthy persons, ${ }^{6}$ whereas A-waves may indicate severely impaired CSF absorption. ${ }^{7-9}$ The origin of these waves is not yet well understood. Auer and Sayama observed rhythmic pial vessel diameter changes correlating with B-waves. ${ }^{10}$ Some authors claim a brain stem centre is responsible for these oscillations. ${ }^{112}$ Others stress the coincidence with respiratory and cardiovascular changes. ${ }^{11-14}$ Transcranial Doppler sonography permits noninvasive measurement of the blood flow velocity in the large basal cerebral arteries. ${ }^{15}$ The diameter of these large arteries is assumed to remain more or less constant. ${ }^{16}$ Changes in cerebral blood flow are caused by constriction or dilatation of the smaller resistance vessels. Therefore, changes of cerebral blood flow velocity in the large basal arteries correlate with changes of cerebral blood flow. ${ }^{17}$ In 5 patients Mautner-Huppert et al showed that middle cerebral artery (MCA) blood flow velocity and ICP were in phase during B-waves. ${ }^{6}$ Goh et al described a decrease in ICP with an increase in MCA blood flow velocity and vice versa during $A$ - and $B$-waves in three hydrocephalic children. In four other children the ICP and TCD signals were in phase. ${ }^{18}$ After subarachnoid haemorrhage, the signals took opposite courses. ${ }^{19}$ After severe head injury, they were in phase. ${ }^{20}$

Whether changes in cerebral blood flow/ volume are the cause or a consequence of changes in ICP, there should be a relationship between spontaneous ICP oscillations and the TCD signal. The following study was designed to assess MCA blood flow velocity and CSF pressure simultaneously in patients with suspected NPH with special emphasis on the characterisation of TCD signal equivalents of spontaneous CSF pressure oscillations.

\section{Materials and methods}

In our institutions patients with suspected NPH who might benefit from shunt surgery are routinely submitted to an overnight recording of ICP. We examined 23 patients $(6$ men, 17 women) with clinical signs of NPH. All patients had ventricular enlargement of various 
degrees on a cranial CT scan and all had at least one of the following symptoms: gait disturbance, dementia, or urinary incontinence. Fourteen patients showed the complete triad of symptoms. In two patients NPH was thought to be secondary to meningitis and subarachnoid haemorrhage, respectively. Age ranged from 55 to 83 years, with the exception of one patient who was 24 years old. The mean age was 69 years. Informed consent was obtained from the patients or their families, or both. In three elderly women we detected either no transcranial Doppler signal or only a weak one which was not adequate for recording. In the remaining 20 patients an overnight (9-18 hours) recording of ICP (in one patient lumbar pressure) and MCA blood flow velocity (9-12 hours, either right or left side) was performed simultaneously.

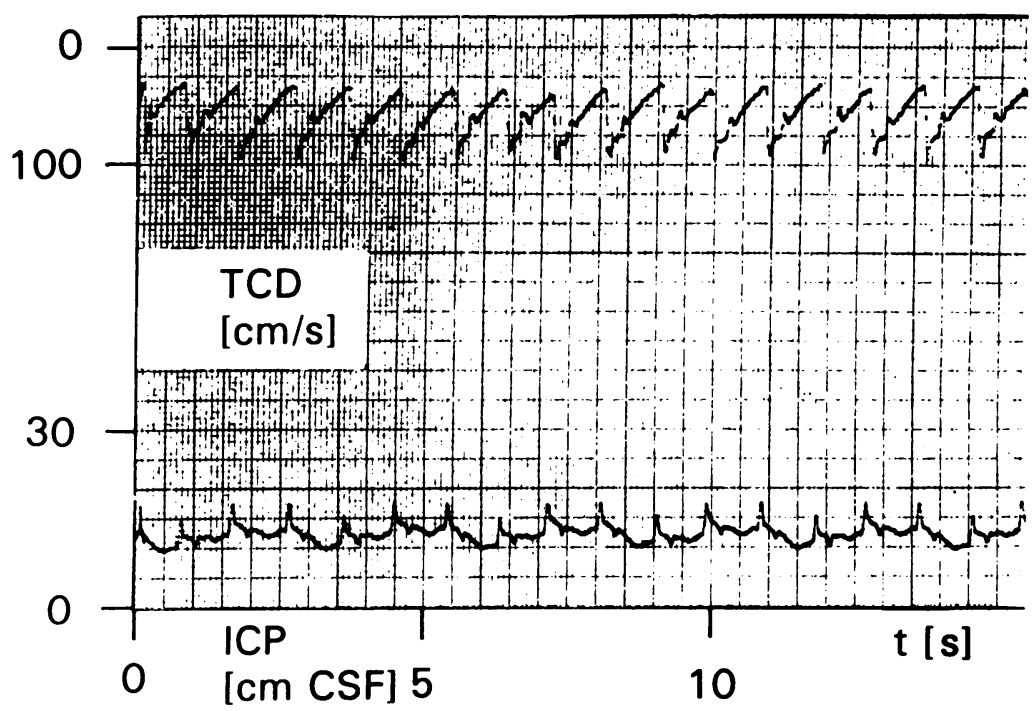

Figure 1 Simultaneous recording of middle cerebral artery blood flow velocity by transcranial Doppler (TCD) and intracranial pressure (ICP). Patient 14. The waveforms are not totally parallel. $A$ rhythm of about 3 cardiac cycles of wavelength is visible in the ICP recording, but not in the TCD recording. There is a slight delay of about $0 \cdot 1 s$ between the ICP and TCD signals during one cardiac cycle.

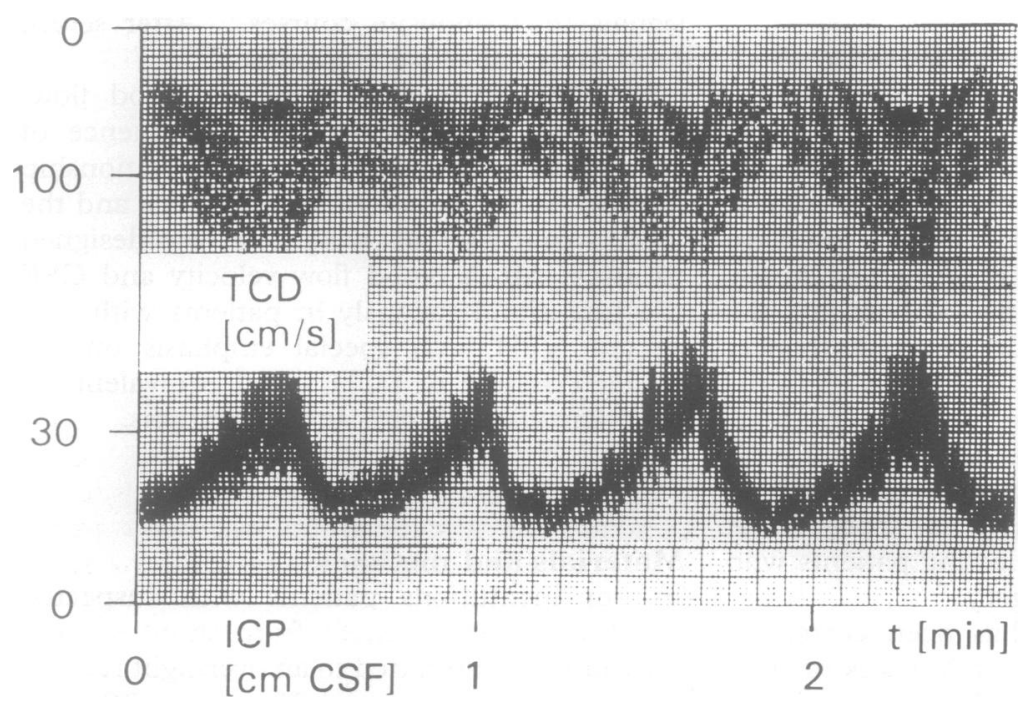

Figure 2 Patient 18. Simultaneous recording of MCA blood flow velocity by TCD and ICP. Permanent B-wave activity. Increase and decrease in the two signals are in phase.
ICP was measured via an intraventricular catheter of $2 \mathrm{~mm}$ diameter inserted into the frontal horn of the left lateral ventricle through a $2-\mathrm{mm}$ burr hole, $2 \mathrm{~cm}$ parasagittal and $2 \mathrm{~cm}$ precoronal, drilled under local anaesthesia. The intraventricular catheter was secured to the scalp with sutures. In one patient CSF pressure was recorded via a lumbar catheter. The transducer (Braun, Germany) was fixed over the bregma and connected to an amplifier (Servomed, Hellige, Germany) and a plotter (Recomed, Hellige, Germany). There were no adverse events such as infection or bleeding. The foramina of Monro were considered to be at $0 \mathrm{~cm} \mathrm{CSF}$. The patients were in a supine position with the head flat.

For the TCD measurements we used the TC 2000S transcranial Doppler (Eden Medizinische Elektronik $\mathrm{GmbH}$, Überlingen, Germany) and recorded only the envelope curve (that is, the maximal velocity), not the entire Doppler spectrum, numerically on the hard disc (sample rate $41 \mathrm{~Hz}$ ). The highest middle cerebral artery blood flow velocity was sought at a depth of 45 to $55 \mathrm{~mm}$ through the temporal window. The side measured was selected dependent on the quality of the signal and interference due to the catheter and sutures. We used the lowest ultrasound intensity still able to produce a satisfactory signal $\left(<100 \mathrm{~mW} / \mathrm{cm}^{2}\right)$. Continuous measurement was made possible by securing the probe in a head ribbon with adhesive tape. A person sat beside the patient for the whole night, checked for any movement of the patient and refastened the TCD probe when necessary. The CSF pressure was recorded throughout the night on a plotter at a speed of $5 \mathrm{~mm} / \mathrm{min}$ and intermittently at a higher speed $(1 \mathrm{~mm} / \mathrm{s}, 10 \mathrm{~mm} / \mathrm{s})$. The transcranial Doppler signal was recorded on the hard disc of the TC $2000 \mathrm{~S}$ and in 15 patients also on the same sheet of paper as the CSF pressure using the second channel of the plotter.

In five patients Doppler signals and CSF pressure were recorded simultaneously during a bolus injection of $10 \mathrm{ml}$ saline via the ventricle catheter.

Sixteen patients had ventriculoatrial or ventriculoperitoneal shunting two to four weeks after the CSF pressure / TCD recording.

\section{Results}

In all patients the initial baseline intracranial / lumbar pressure was less than $20 \mathrm{~cm} \mathrm{CSF}$, which was consistent with a diagnosis of "normal" pressure hydrocephalus. The Doppler recording was more susceptible to movement artefacts than the pressure registration. However, the artefacts could be reliably identified. In the 15 parallel recordings the course of the Doppler signal was always almost parallel to and in phase with the course of the CSF pressure.

At a speed of $10 \mathrm{~mm} / \mathrm{s}$ during one cardiac cycle, there was only a delay of about $0 \cdot 1 \mathrm{~s}$ between the systolic peak and the dicrotic incisura of the CSF pressure and of the blood 

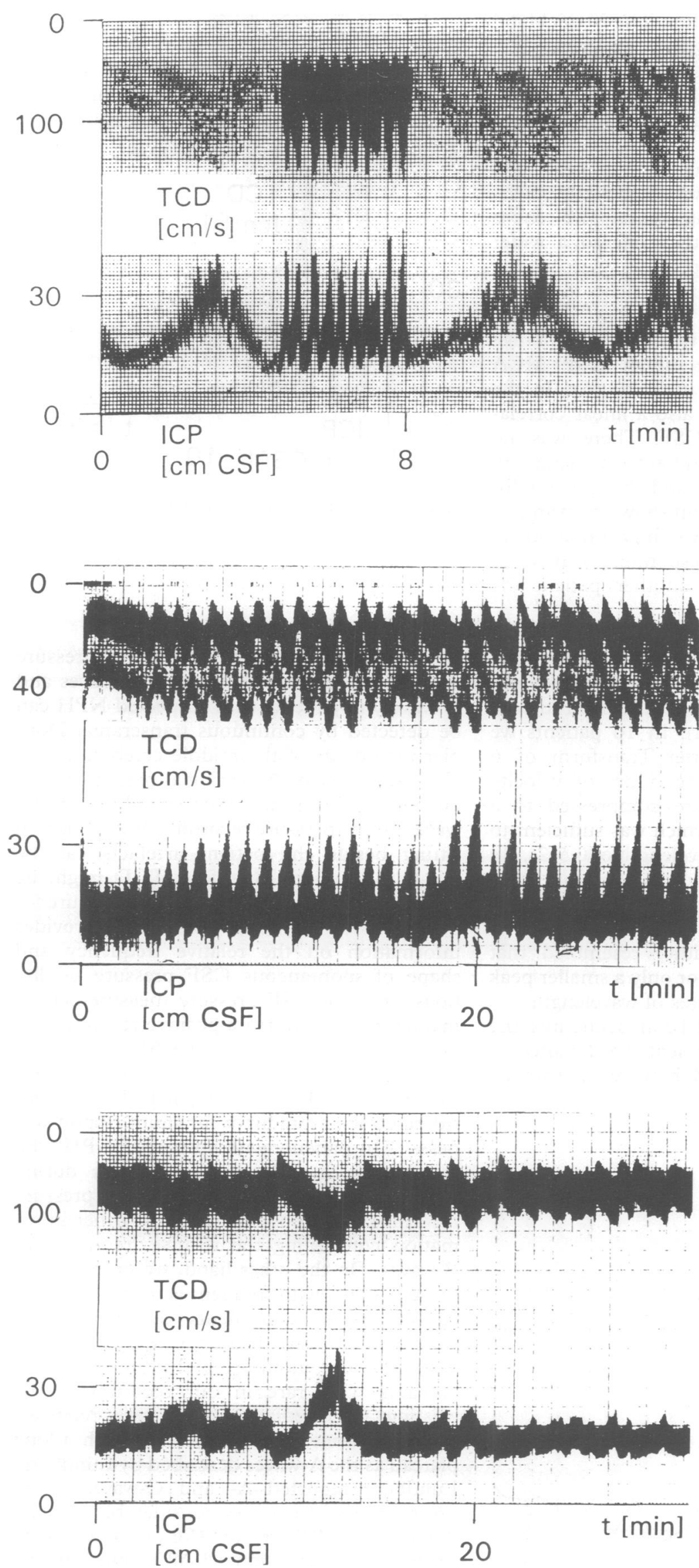

Figure 3 Simultaneous recording of MCA blood flow velocity by TCD and ICP in three different patients.

3a (top): Permanent B-wave activity. Speed was changed from $1 \mathrm{~mm} / \mathrm{s}$ to $5 \mathrm{~mm} / \mathrm{min}$ and back to $1 \mathrm{~mm} / \mathrm{s}$. Same patient as in fig. 2 (patient 18).

$3 b$ (middle): Patient 4. Permanent B-wave activity.

$3 c$ (bottom): Patient 14. Several B-waves and an intermediate wave. C-waves on top of the intermediate wave. flow velocity (fig 1 ). The waveform was slightly different. A rhythm with a wavelength of about three heartbeats was seen in the pressure recording, but not in the Doppler recording (probably corresponding to respiration).

The two recordings were also almost parallel at a speed of $1 \mathrm{~mm} / \mathrm{s}$. This speed provides a good image of the B-waves and their TCD equivalents in detail (fig 2 ). The routinely used speed of $5 \mathrm{~mm} / \mathrm{min}$ is appropriate for visualising intermediate waves (see below) and long runs of B-waves and their TCD equivalents (figures $3 a, b, c$ ).

In all of the 20 patients we observed B-waves with various wavelengths and amplitudes having sine-typed or ramp-typed shape. The relative frequency was variable. In four patients there were a few waves lasting longer than 2 minutes and 40-60 cm CSF high. These latter waves (considered as intermediate waves, between A- and B-waves) were clearly distinguishable from the surrounding smaller B-waves, however, they never reached a plateau of several minutes as the A-waves described by Lundberg. ${ }^{5}$ Some of them looked like large ramp-typed B-waves, others resembled A-waves with only a short plateau. C-waves were observed in only two patients (fig 3c). To confirm the visual impression of an almost parallel course of CSF pressure and MCA blood flow velocity, we performed measurements at this speed $(5 \mathrm{~mm} / \mathrm{min})$ in each of the 15 patients (ICP and TCD on one sheet of paper) of the systolic value of the peak and the diastolic value of the following dip in 5 TCD B-wave equivalents (and not knowing that there also is a B-wave in the pressure recording). Then we measured the corresponding values of the pressure recording. We did the same for 5 other B-waves seen first on the pressure recording and then measured the corresponding Doppler values. In all of these 150 B-waves/B-wave equivalents maximum and minimum values were reached in both recordings at the same time. There was no $\mathrm{B}$-wave recording that was not reflected by a $B$-wave equivalent in the TCD and vice versa. We also measured $2 \times 11$ intermediate waves and their TCD equivalents in four patients and observed the same result. Intermediate waves and their TCD equivalents were higher than $B$-waves and their TCD equivalents in the same patient. TCD artefacts due to movement with probe displacement were reliably detected. In two restless patients who produced frequent artefacts, some of these artefacts (for example, due to yawning, micturition, or coughing) resembled spontaneous ICP oscillations (probably due to pressing and subsequent rise of intrathoracic and venous pressure) and were not correlated with changes in MCA blood flow velocity. The occurrence of these artefacts was noted by the night watch.

There was no difference between the lumbar and ventricular pressure measurements for spontaneous CSF pressure oscillations and their TCD equivalents.

In each of the four patients with intermediate waves we made one graph with 10 B-waves and 1-10 intermediate waves sampled 
randomly from the recording. The MCA blood flow velocity constituted the one coordinate, the CSF pressure the other. Each wave consisted of two points, the peak (systolic value) and the following dip (diastolic value), as these parameters were easily measurable in the recording. Diastolic value of the peak and systolic value of the dip were frequently superimposed by surrounding values. We chose these four patients, as they offered a wider range of velocity and pressure due to the intermediate waves. In three patients there was a good intraindividual correlation of CSF pressure and TCD signal within a certain range $(0-30 \mathrm{~cm} \mathrm{CSF})$. Above $30 \mathrm{~cm}$ CSF MCA blood flow velocity rose more slowly. In the fourth patient there was also a linear correlation above $30 \mathrm{~cm}$ of CSF. There was no interindividual correlation between changes in MCA blood flow velocity and changes in CSF pressure. One patient might show, for example, B-wave equivalent activity of high amplitude in the TCD recording, corresponding to $\mathrm{B}$-waves of low amplitude, and another patient the opposite.

With the TC $2000 \mathrm{~S}$ it is possible to create ASCII "export" files off line that include the values of the envelope curve (41 values per second) or the mean of the values next to each other (data compression). In 16 patients we performed a Fast Fourier Transform of 6 continuous early morning hours of velocity recording. The data were compressed to a sample rate of $41 / \mathrm{min}$, which was sufficient to detect B-waves with a wavelength of $0 \cdot 5-2 \mathrm{~s}$. We show a representative power spectrum of a patient with almost permanent B-wave activity (fig 4).

The other spectra either resembled that shown in fig 4 or had no or only a smaller peak between 0.5 and 2 minutes of wavelength.

The bolus injection of $10 \mathrm{ml}$ saline into the lateral ventricle of five patients hardly affected the Doppler signal. It did, however, elevate the ICP considerably (fig 5 ).

spectral

power

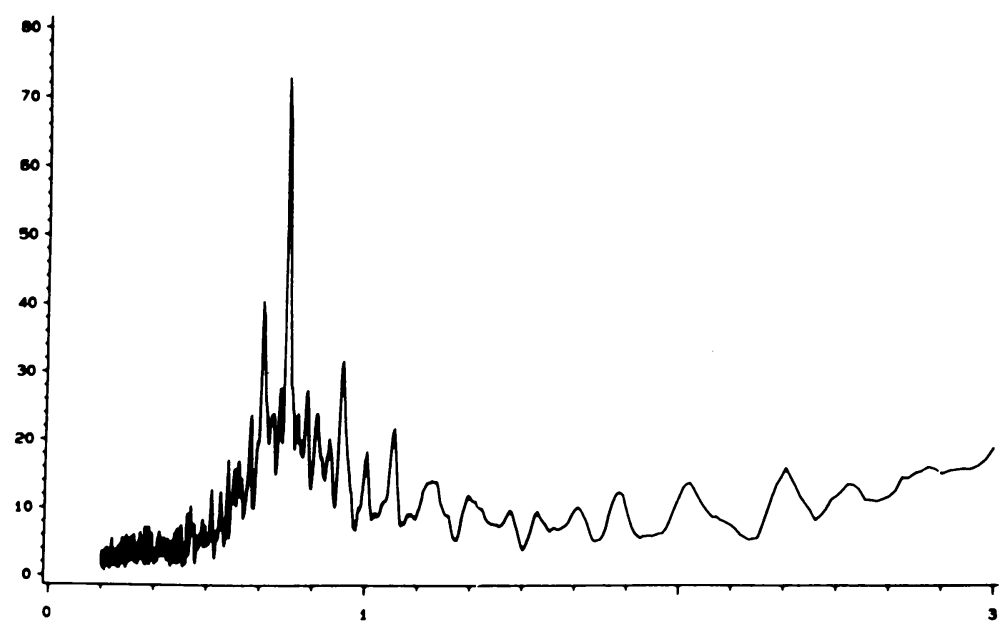

wavelength [min]

Figure 4 Fast Fourier Transform of the transcranial Doppler recording over 6 hours. $A$ peak of the spectral power at 40-50s corresponding to almost permanent B-wave activity in this patient (patient 3).

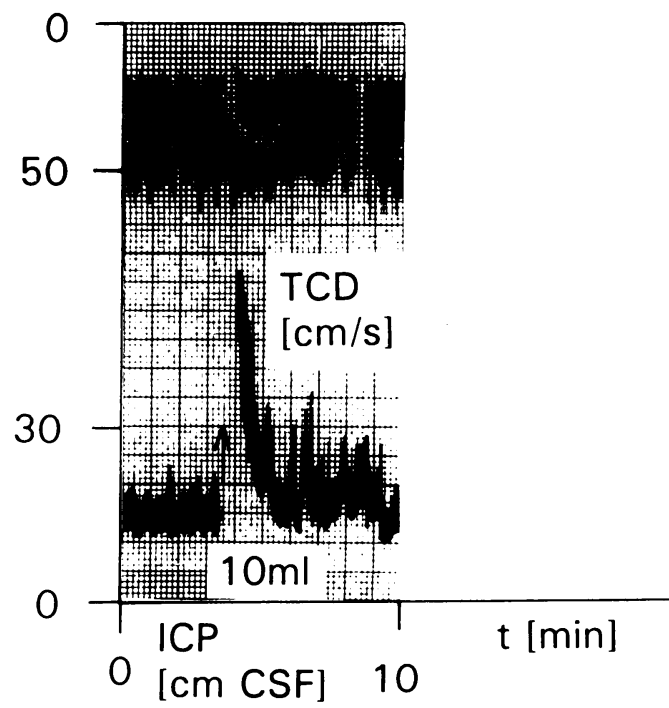

Figure 5 Simultaneous recording of MCA blood flow velocity by TCD and ICP. Patient 17 . Injection of $10 \mathrm{ml}$ saline into the lateral ventricle (arrow) raised the ICP considerably, but hardly affected the TCD signal.

\section{Discussion}

Equivalents of spontaneous CSF pressure oscillations (B-waves, intermediate waves and C-waves) in patients with suspected NPH can be detected by continuous transcranial Doppler recordings of the middle cerebral artery. Conversely, forms appearing as pressure waves on a TCD recording correspond to similar waves on the pressure recording. With a person sitting beside the patient artefacts can be reliably identified. Therefore, TCD might be useful as a noninvasive screening procedure for patients with suspected NPH, as it provides information on the relative frequency and shape of spontaneous CSF pressure oscillations. Invasive CSF pressure measurement is mandatory to confirm a positive result and to assess the absolute level of CSF pressure at baseline and during oscillations. The relative frequency of B-waves, in particular, is considered to be a predictor for shunt responsiveness. ${ }^{1-4}$ In patients with suspected NPH who show only few B-wave equivalents during continuous TCD recording, CSF pressure recordings may possibly give no further information concerning the relative frequency of B-waves. On the other hand, we propose that those patients with frequent activity of B-wave equivalents or equivalents of intermediate waves should have further investigation with CSF pressure monitoring. Intermediate and $\mathrm{C}$-waves were rare in our recordings. Any existing intermediate waves were relatively small in wavelength and did not reach a long plateau as the A-waves described by Lundberg. Lundberg reported $\mathrm{A}$ - and $\mathrm{C}$-waves to be present in patients with a considerable increase in the mean CSF pressure (for example, due to space-occupying lesions)..$^{5}$ All of our patients had "normal" pressure hydrocephalus.

Three of our patients initially showed a linear correlation between TCD signal and CSF pressure, however, at levels above approximately $30 \mathrm{~cm}$ CSF with increasing pressure the concomitant increase in blood flow velocity 
was less pronounced. In another patient there seemed to be a linear correlation above $30 \mathrm{~cm}$ CSF, too.

There was an almost parallel increase and decrease in the two signals during spontaneous oscillations. This is consistent with the findings reported by Mautner-Huppert et al, ${ }^{6}$ Goh et $a l^{18}$ and Newell et al. ${ }^{20}$ The absence of marked MCA blood flow velocity changes during injection of normal saline into the lateral ventricle with a concomitant increase in CSF pressure supports the hypothesis that spontaneous CSF pressure oscillations are of vasogenic origin. The origin of vasodilatation at the beginning of a $\mathrm{B}$-wave and of vasoconstriction at the end of a B-wave as described by Auer and Sayama ${ }^{9}$ and their relation (cause or effect) to raised intracranial pressure remain unclear. There are different opinions on the role of rhythmic hyperventilation and hypoventilation (and subsequent changes in $\mathrm{pCO}_{2}$ and vessel diameter, for example, in CheyneStokes respiration), neurogenic vasodilatation and vasoconstriction, and changes in blood pressure and heart rate. Lundberg ${ }^{4}$ and Maeda et $a l^{21}$ reported that A-waves appear not only during spontaneous, but also during artificial respiration, but that B-waves are often effaced with the start of artificial ventilation. Shima et $a l^{22}$ described hypoventilation during plateau waves in humans. Changes in heart rate were minimal. Newell et al ${ }^{20}$ found B-waves in artificially ventilated patients. Hashimoto et $a l^{14}$ described apnoea at the beginning of a B-wave, followed by respiration and increasing heart rate and blood pressure: the peak of a B-wave was reached before the peak of heart rate and blood pressure. Borgesen and Espersen $^{23}$ found no correlation between arterial $\mathrm{pCO}_{2}$ and $\mathrm{A}$ - and $\mathrm{B}$-waves, excluding changes in respiration to be a causative factor. Einhüpl et $a l^{13}$ reported hyperventilation to coincide with the B-wave peak in patients with and without Cheyne-Stokes respiration. Animal experiments conducted by Maeda $e t a l^{21}$ and Kogure et al ${ }^{12}$ using electrical stimulation showed evidence of a brainstem centre being responsible for neurogenic vasodilatation and vasoconstriction. These findings, however, are difficult to interpret with regard to the cause of spontaneous ICP oscillations, as electrical stimulation is non-physiological. The fact that ICP rises in sleep stage II and in REM sleep and the fact that spontaneous ICP oscillations are more frequent in REM sleep ${ }^{182425}$ suggests that spontaneous CSF pressure oscillations are of cerebral origin mediated by changes in vessel diameter.

The Fast Fourier Transform of a TCD signal over 6 hours is helpful, since the frequency and amplitude of B-wave equivalents are taken into account. As shunting in NPH results in a reduction of the amplitude and the relative frequency of B-waves, ${ }^{26}$ TCD and Fast Fourier Transform might also be useful in controlling the result of shunting.

We are very grateful to Eden Medizinische Elektronik GmbH Uberlingen, Germany for supplying us with the TC $2000 \mathrm{~S}$ an to Professor Schulte-Mönting for the biomathematical interpretation of the data.
1 Symon L, Dorsch NWC. Use of long-term intracranial pressure measurement to assess hydrocephalic patients prior to shunt surgery. $\mathcal{f}$ Neurosurg $1975 ; 42: 258-73$.

2 Pickard JD. Adult communicating hydrocephalus. $\mathrm{Br} \mathcal{f}$ Hosp Med 1982;27:35-44.

3 Black PM, Ojemann RG, Tzouras A. CSF shunts for dementia, incontinence, and gait disturbance. Clin Neurosurg 1985;32:632-51.

4 Graff-Radford NR, Godersky JC, Jones MP. Variables predicting outcome in symptomatic hydrocephalus in the elderly. Neurology 1989;39:1601-4.

5 Lundberg $\mathrm{N}$. Continuous recording and control of ventricular fluid pressure in neurosurgical practice. Acta Psychiatr Neurol Scand 1960;Suppl 149:1-193.

6 Mautner D, Dirnagl U, Haberl R, Schmiedeck P, Garner C, Villringer A, Einhäupl KM. B-waves in healthy persons. In: Hoff JT, Betz AL, eds. Intracranial pressure VII. Berlin, Heidelberg: Springer-Verlag, 1989:209-12

7 Hayashi M, Kobayashi H, Kawano H, Handa Y, Yamamoto $S$, Kitano T. ICP patterns and isotope cisternography in patients with communicating hydrocephalus following patients with communicating hydrocephalus following rupture

8 Yokota A, Matsuoka S, Ishikawa T, Kohshi H, Kajiwara H. Overnight recording of intracranial pressure and electroencephalography in neurosurgical patients. Part I: Intracranial pressure waves and their clinical correlations (abstract). Sangyo Ika Daigaku Zasshi 1989;11:371-81.

9 Hayashi M, Handa Y, Kobayashi H, Kawano H, Ishii H, Hirose S. Plateau waves and CSF circulation. The eighth international symposium on intracranial pressure (Abst 202). Rotterdam. 16-20 June 1991. Abstract 202.

10 Auer LM, Sayama I. Intracranial pressure oscillations (B-waves) caused by oscillations in cerebrovascular volume. Acta Neurochir 1983;68:93-100.

11 Higashi S, Yamamoto S, Hashimoto M, Fujii $\mathrm{H}$, Ito $\mathrm{H}$ Kogure Y, Tokuda $\mathrm{K}$. The role of vasomotor center and adrenergic pathway in B-waves. In: Hoff JT, Betz AL, eds. Intracranial pressure VII. Berlin, Heidelberg: SpringerIntracranial pressure

12 KogureY, Fujii H, Higgashi S, Hashimoto M, Tokuda K, Ito H, Yamamoto S. Electroencephalographic changes during plateau waves induced by electrical stimulation of the brain stem. In: Hoff JT, Betz AL, eds. Intracranial pressure VII. Berlin, Heidelberg: Springer-Verlag, 1989:235-7.

13 Einhäupl KM, Garner C, Dirnagl U, Schmiedeck P, Kufner $G$, Rieder J. Oscillations of ICP related to cardiovascular parameters. In: Miller JD, Teasdale GM, Rowan JO, Galbraith S, Mendelow AD eds. Intracranial pressure VI. Berlin, Heidelberg: Springer-Verlag, 1986:290-7.

14 Hashimoto M, Higashi S, Kogure Y, Fujii H, Tokuda K, Ito $H$, Yamamoto $S$. Respiratory and cardiovascular oscillations during B-waves. In: Hoff JT, Betz AL, eds. Intracranial pressure VII. Berlin, Heidelberg: SpringerIntracranial pressure

15 Aaslid R, Markwalder TH, Nornes H. Noninvasive transcranial Doppler ultrasound recording of flow velocity in basal cerebral arteries. $\mathcal{f}$ Neurosurg 1982;57:769-74.

16 Huber P, Handa J. Effect of contrast material, hypercapnia, hyperventilation, hypertonic glucose and papaverine on the diameter of the cerebral arteries. Invest Radiol 1967;2:17-32.

17 Bishop CCR, Powell S, Rutt D, Browse NL. Transcranial Doppler measurement of middle cerebral blood flow velocity. A validation study. Stroke 1986;17:913-5.

18 Goh D, Minns RA, Pye S, Steers J. Continuous intraventricular pressure and simultaneous cerebral blood flow velocity (CBFV) measurements during sleep in velocity (CBFV measurements during sleep in hydrocephalic children. The eighth international symposium on
intracranial pressure (Abst 221). Rotterdam, 16-20 June intracrani.

19 Hashimoto T, Nakamura N, Kanki T, Abe S. ICP and flow dynamics after subarachnoid haemorrhage. The eighth
international symposium on intracranial pressure (Abst 109). Rotterdam, 16-20 June 1991 .

20 Newell DW, Stooss R, Aaslid R, Reulen HJ. Spontaneous fluctuations in cerebral blood flow as a cause of $B$-waves. The eighth international symposium on intracranial pressure (Abst 127). Rotterdam, 16-20 June 1991.

21 Maeda M, Matsuura S, Tanaka K, Katsuyama J, Nakamura H, Sakamoto H, Nishimura S. Effects of electrical stimulation on intracranial pressure and systemic arterial blood pressure in cats. Part I. Stimulation of brain stem. Neurol Res 1988;10:87-92

22 Shima K, Ueno K, Chigaski H, Ishii S. Comparative study with experimental and clinical plateau waves relating to sleep. In: Hoff JT, Betz AL, eds. Intracranial pressure VII. sleep. In: Hoff JT, Betz AL, eds. Intracranial press

23 Borgesen SE, Espersen JO. The correlation between A- and B-waves and arterial $\mathrm{CO}_{2}$ tension. In: Miller JD, Teasdale B-waves and arterial $\mathrm{CO}_{2}$ tension. In: Miller JD, Teasdale
$\mathrm{GM}$, Rowan JO, Galbraith $\mathrm{S}$, Mendelow $\mathrm{AD}$, eds. Intracranial pressure VI. Berlin, Heidelberg: SpringerIntracranial pressure $V$

24 Ogashiwa $M$, Takeuchi $K$. Intracranial pressure changes during sleep in man. No To Shinkei 1983;35:123-9.

25 Yokota A, Matsuoka S, Ishikawa T, Kohshi K, Kajiwara H. Overnight recordings of intracranial pressure and electroencephalography in neurosurgical patients. Part II: Changes in intracranial pressure during sleep. Sangyo Ika Daigaku Zasshi 1989;11:383-91.

26 Tanaka K, Nishimura S. The importance of outflow resistance of the shunt system for elimination of B-waves. In: Hoff JT, Betz AL, eds. Intracranial pressure VII. Berlin, Heidelberg: Springer-Verlag, 1989:368-73. 